\title{
Effect of impedance variation around the fundamentals on PA distortions characteristics under wideband multi-tone stimulus
}

\begin{abstract}
Characterisation of modern wireless power transistors and amplifies requires widebandmodulated stimuli for realistic performance evaluation. Normally power transistor performance is evaluated using passive load-pull techniques. However, these have some physical realization constraints that influence the amplitude and the phase of the presented impedance at the DUT plane as across the modulated bandwidth. The problem becomes more apparent as modulation bandwidth is increased beyond few MHz. It has been shown recently that a digitally controlled active envelop load-pull (ELP) system can completely address this problem [1], thus allowing for a more systematic investigation of the parameters that may affect the performance of power transistors under wideband-modulated stimuli. In this paper, non ideal multi-tone impedance conditions are purposely introduced for demonstrating their effects on power amplifier performance in order to illustrate the DUT sensitivity to measurement system imperfections at higher modulation bandwidths in excess of few MHz.
\end{abstract}

Keyword: Power amplifier; Non-linear; Multi-tone 